\title{
Sound Transmission Loss Prediction of the Composite Fuselage with Different Methods
}

\author{
Chongxin Yuan • Otto Bergsma • Adriaan Beukers
}

Received: 28 January 2011 /Accepted: 30 March 2011 /Published online: 15 April 2011

(C) The Author(s) 2011. This article is published with open access at Springerlink.com

\begin{abstract}
Increase of sound transmission loss(TL) of the fuselage is vital to build a comfortable cabin environment. In this paper, to find a convenient and accurate means for predicting the fuselage TL, the fuselage is modeled as a composite cylinder, and its TL is predicted with the analytical, the statistic energy analysis (SEA) and the hybrid FE\&SEA method. The TL results predicted by the three methods are compared to each other and they show good agreement, but in terms of model building the SEA method is the most convenient one. Therefore, the parameters including the layup, the materials, the geometry, and the structure type are studied with the SEA method. It is observed that asymmetric laminates provide better sound insulation in general. It is further found that glass fiber laminates result in the best sound insulation as compared with graphite and aramid fiber laminates. In addition, the cylinder length has little influence on the sound insulation, while an increase of the radius considerably reduces the TL at low frequencies. Finally, by a comparison among an unstiffened laminate, a sandwich panel and a stiffened panel, the sandwich panel presents the largest TL at high frequencies and the stiffened panel demonstrates the poorest sound insulation at all frequencies.
\end{abstract}

Keywords Sound transmission loss $\cdot$ SEA $\cdot$ Fuselage $\cdot$ Cylinder

\section{Introduction}

Acoustic transmission is one of the principal design drivers for composite fuselages [1]. Therefore the prediction of sound transmission loss (TL) of a fuselage structure is of major importance. There are two major noise sources when the airplane is in flight; one is the turbulent boundary layer noise and the other is the engine noise. The engine noise can also be divided into airborne noise and structure-born noise. In this study only the airborne noise is considered as the noise source.

C. Yuan $(\bowtie) \cdot$ O. Bergsma $\cdot$ A. Beukers

Department of Production Technology, Faculty of Aerospace Engineering,

Delft University of Technology, Delft, The Netherlands

e-mail: yuanchongxin@yahoo.com.cn 
For the airborne noise from the engine, a large number of investigations have been devoted to the prediction of fuselage TL. In these studies the fuselage structure was usually idealized as the cylinder. The published models mainly considered three classical types of constructions: monolithic, stiffened and sandwich. Koval [2] studied the TL of infinite isotropic cylinders considering the external airflow and internal shell pressurization. The impedance of the shell and its content were used to determine the TL. He also developed a theory to investigate the TL of orthotropic and laminated composite shells [3, 4]. A program to predict the noise transmission into aircraft was developed by ESDU [5]. In this program, the noise excitation included single frequency plane waves and broadband noise in a diffused field. The structure was assumed as an eccentrically stiffened cylinder with isotropic materials. Yvette Y. Tang [6] predicted the TL through a cylindrical sandwich shell with honeycomb core. In his paper, the thin shell theory and the first order shell theory were compared to each other. For the first order shell theory, the shear and rotation effects were taken into account. Results show that the shear waves transmit sound through the shell, resulting in a decrease of TL in high frequencies. K. Daneshjou et al. [7, 8] combined the work of Koval and Yvette to generate two more explicit analytical models with thin shell and first order theory respectively. Besides the analytical models, some commercial software has also been used to predict the TL of more complex structures. Rajesh Arjunan [9] performed a parametric analysis of curved honeycomb composites panels with the software "Va one" in his master thesis. Zhuang Li [10] made a TL prediction on sandwich composites with AutoSEA, whereby a two-room method experiment is performed to verify the modeling results. The predicted and measured results show good agreement.

The transmission loss of elastic structures can be numerically estimated using finite element methods (FE), boundary element methods (BEM) and statistic energy analysis (SEA). FE and BEM can model the vibro-acoustic behavior of a structure more accurately compared to SEA. However they require extensive computational resources in the case of large structures and high frequencies. In contrast, the SEA method can be more convenient and rapid in the case of large structures so it is often used to model the sound transmission. In the SEA method, the structure is divided into different subsystems. For example, a fuselage consists of crown panels, side panels and keel panels, windows, and frames subsystems. Each subsystem can also be represented by three parts: one represents out-ofplane motion and the other two represent in-plane stretching and shear motion respectively. For each subsystem, the principle of energy conservation must be abided [11]. There are two approaches for applying the SEA methodology [12]. The first, "mode approach", is based on modeling each subsystem as resonant responses. Most of the references mentioned here have adopted this method. The second, "wave approach", is based on modeling each subsystem as a superposition of waves travelling throughout the subsystem [13-15].

In this paper, an analytical model regarding an infinite-length composite cylinder is built. Next, a SEA model and a hybrid FE\&SEA model are used to predict the TL of a limitedlength cylinder. The TL results computed by these three models are compared to each other. Finally, due to its efficiency, the SEA model is used for studying the effect of different parameters on the fuselages TL.

\section{Analytical Model}

As shown in Fig. 1, it is assumed that a plane wave with incident angle $\gamma$ hits the cylinder. The cylinder radius is $1,975 \mathrm{~mm}$ and the length is infinite. Because of the cylindrical structure, all analyses are conducted in cylindrical coordinates $\{z, \varphi, r\}$ denoting axial, 
circumferential and radial direction respectively. The properties of the Carbon/Epoxy materials used here are:

$E_{1}=221 \mathrm{GPa}, E_{2}=6.9 \mathrm{GPa}, G_{12}=4.8 \mathrm{GPa}, v_{12}=0.25, \rho=1600 \mathrm{~kg} / \mathrm{m}^{3}, t_{\mathrm{ply}}=0.159 \mathrm{~mm}$

The original laminates layup is $[0 / 90 / 45 /-45 / 0]_{\mathrm{s}}, c_{1}, c_{2}$ are the sound speed in the cabin air and the ambient air, and $\rho_{1}, \rho_{2}$ are the densities of the cabin air and the ambient air respectively. And $c_{1}=c_{2}=343 \mathrm{~m} / \mathrm{s}, \rho_{1}=\rho_{2}=1.29 \mathrm{~kg} / \mathrm{m}^{3}$.

The thin shell theory is used to build a mathematical model based on the following assumptions:

1. The ratio of the shell thickness to the cylinder radius is much smaller than 1 ;

2. The effect of rotational inertia is negligible;

3. The layer deformation complies with the Kirchhoff hypothesis;

4. There is no relative displacement at the interface of layers;

\subsection{The Sound Transmission Loss}

The sound transmission loss (TL) of the shell is defined as the ratio of the transmitted power $\mathrm{W}^{\mathrm{T}}$ and the incident power $\mathrm{W}^{\mathrm{I}}$ per unit length of the cylinder:

$$
T L=10 \log _{10}\left(\frac{W^{I}}{W^{T}}\right)
$$

where,

$$
\begin{gathered}
W^{\mathrm{I}}=\frac{\cos (\gamma) \cdot P_{0}^{2}}{\rho_{1} c_{1}} \cdot R \\
W^{\mathrm{T}}=\frac{1}{2} \cdot \operatorname{Re}\left\{\int_{0}^{2 \pi} P_{2}^{\mathrm{T}} \cdot \frac{\partial}{\partial t}(w)^{*} r \mathrm{~d} \varphi\right\}, r=R
\end{gathered}
$$

in which $\gamma$ is incident angle of acoustic wave, $P_{0}$ is the amplitude of the incident wave (constant value), $R$ is the radius of the cylinder, $\rho_{1}$ and $c_{1}$ are density and sound speed of the external air. $P_{2}^{\mathrm{T}}$ is the transmitted wave pressure as a function of time and frequency, $\varphi$ the

Fig. 1 Schematic diagram of incident acoustic plane wave onto the fuselage

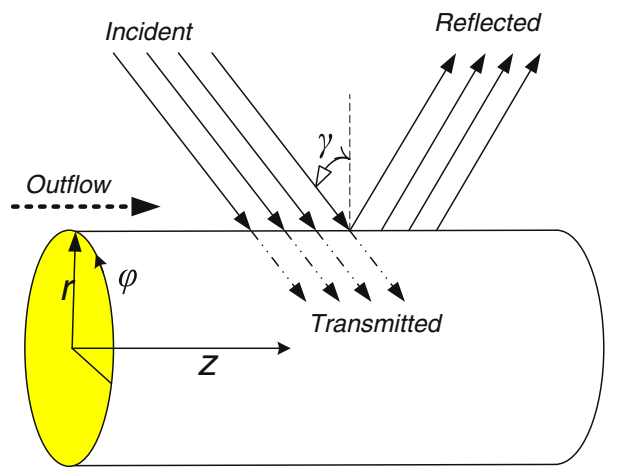


angle around the cross section (Fig. 1), $w$ the shell displacement in radial direction. The superscript * denotes the complex conjugate of the argument.

As can be seen in Eqs. 1-3, the unknown $P_{2}^{\mathrm{T}}$ and $w$ should be given to predict the transmission loss. Their expressions will be discussed in the following section.

\subsection{The Governing Equation of Sound Wave}

The governing equations of sound waves are dissimilar for the inside and outside of cylinders. These governing equations lead to the expression for the acoustic pressure and the full set of shell displacements.

\subsubsection{Inner Cavity}

For the internal cavity of the cylinder, it is supposed that the shell interior is totally absorptive and we assume that only an inward traveling wave exists [6]. The acoustic pressure of the inner cavity satisfies:

$$
c_{2}^{2} \nabla^{2} p_{2}^{T}-\frac{\partial^{2}}{\partial t^{2}} p_{2}^{T}=0
$$

where, $\nabla^{2}=\frac{1}{r} \frac{\partial}{\partial r}\left(r \frac{\partial f}{\partial r}\right)+\frac{1}{r^{2}} \frac{\partial^{2} f}{\partial \varphi^{2}}+\frac{\partial^{2} f}{\partial z^{2}}$, is the Laplace operator expressed in cylindrical coordinates, and $c_{2}$ is the sound speed in the cylinder.

\subsubsection{Outer Cavity}

Because the airflow outside the cylinder is a combination of the incident wave and the reflected wave, the outflow of the cylinder (the external flow and the reflected sound wave) must satisfy the convected wave equation [6]:

$$
c_{1}^{2} \nabla^{2}\left(p_{1}^{I}+p^{R}\right)-\left(\frac{\partial}{\partial t}+\mathbf{V} \cdot \nabla\right)^{2}\left(p_{1}^{I}+p_{1}^{R}\right)=0
$$

in which $\mathbf{V}$ is the velocity of the external airflow, where $\mathbf{V}=<V_{\mathrm{x}}, V_{\mathrm{y}}, V_{\mathrm{z}}>$. In this case, because the external airflow propagates in axial direction, $V_{\mathrm{x}}=0, V_{\mathrm{y}}=0 . p_{1}^{\mathrm{I}}$ is the pressure of the incident wave and $p_{1}^{\mathrm{R}}$ is the pressure of the reflected wave.

\subsection{Equations of Motion}

The equations of motion are given here for expressing the shell displacements as a function of time [4]:

$$
\begin{gathered}
\frac{\partial}{\partial z} N_{z}+\frac{1}{R} \cdot \frac{\partial}{\partial \varphi} N_{z \varphi}+q_{z}=I \frac{\partial^{2}}{\partial t^{2}} u \\
\frac{1}{R} \frac{\partial}{\partial \varphi} N_{\varphi}+\frac{\partial}{\partial z} N_{z \varphi}+\frac{1}{R}\left(\frac{1}{R} \frac{\partial}{\partial \varphi} M_{\varphi}+\frac{\partial}{\partial z} M_{z \varphi}\right)+q_{\varphi}=I \frac{\partial^{2}}{\partial t^{2}} v \\
-\frac{N_{\varphi}}{R}+\frac{\partial^{2}}{\partial z^{2}} M_{z}+\frac{2}{R} \frac{\partial^{2}}{\partial z \partial \varphi} M_{z \varphi}+\frac{1}{R^{2}} \frac{\partial}{\partial \varphi} M_{\varphi}+q_{r}=I \frac{\partial^{2}}{\partial t^{2}} w
\end{gathered}
$$


where $q_{\mathrm{z}}, q_{\varphi}, q_{\mathrm{r}}$ are the external forces per unit area in the axial, circumferential and radial directions respectively. $N$ and $M$ are the forces and moment resultants. $u, v, w$ are the shell displacements of the neutral face in the axial, circumferential and radial directions respectively.

$I$ is the mass inertia and can be expressed by:

$$
I=\sum_{k=1}^{N} \int_{\xi_{[k-1]}}^{\xi_{[k]}} \rho \mathrm{d} x
$$

where $\rho$ is the density per layer and $\xi_{\mathrm{f}}$ is the centroid line position of the $k_{\mathrm{th}}$ layer (Fig. 2). In this circumstance, because the pressure is varies only in the radial direction, $q_{\mathrm{z}}=q_{\varphi}=0, q_{\mathrm{r}}=p_{1}^{\mathrm{I}}+p_{1}^{\mathrm{R}}-p_{3}^{\mathrm{T}}$. The expressions for the incident wave pressure $P_{1}^{\mathrm{I}}$, the reflected wave pressure $P_{1}^{\mathrm{R}}$ and the transmitted wave pressure $P_{3}^{\mathrm{T}}$ will be introduced later.

The forces $(N)$ and moments $(M)$ relations can be calculated by:

$$
\left[\begin{array}{l}
N_{\mathrm{z}} \\
N_{\varphi} \\
N_{\mathrm{z} \varphi} \\
M_{\mathrm{z}} \\
M_{\varphi} \\
M_{\mathrm{z} \varphi}
\end{array}\right]=\left[\begin{array}{ll}
\mathbf{A} & \mathbf{B} \\
\mathbf{B} & \mathbf{D}
\end{array}\right] \cdot\left[\begin{array}{l}
\varepsilon_{\mathrm{z}} \\
\varepsilon_{\varphi} \\
\gamma_{\mathrm{z} \varphi} \\
k_{\mathrm{z}} \\
k_{\varphi} \\
k_{\mathrm{z} \varphi}
\end{array}\right]
$$

where:

$$
\begin{gathered}
\mathbf{A}=\sum_{k=1}^{\mathrm{N}} \mathbf{Q}_{[k]}\left(\xi_{[k]}-\xi_{[k-1]}\right) \\
\mathbf{B}=\frac{1}{2} \sum_{k=1}^{\mathrm{N}} \mathbf{Q}_{[k]}\left(\xi_{[k]}^{2}-\xi_{[k-1]}^{2}\right) \\
\mathbf{D}=\frac{1}{3} \sum_{k=1}^{\mathrm{N}} \mathbf{Q}_{[k]}\left(\xi_{[k]}^{3}-\xi_{[k-1]}^{3}\right)
\end{gathered}
$$

Fig. 2 Laminates layup

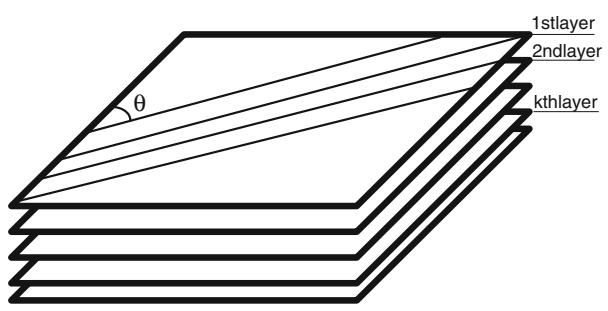


$\mathbf{Q}_{[\mathbf{k}]}$ are the transformed stiffness matrices [16] for the laminated layers with different fiber angles $\theta$ (Fig. 2). The strains and curvatures for Eq. 10 can be written as [17]:

$$
\begin{gathered}
\varepsilon_{z}=\frac{\partial}{\partial z} u ; \varepsilon_{\varphi}=\frac{1}{R}\left(\frac{\partial}{\partial \varphi} v+w\right) ; \gamma_{z \varphi}=\frac{\partial}{\partial z} v+\frac{1}{R} \frac{\partial}{\partial \varphi} u \\
k_{z}=-\frac{\partial^{2}}{\partial z^{2}} w ; k_{\varphi}=\frac{1}{R^{2}}\left(\frac{\partial}{\partial \varphi} v-\frac{\partial^{2}}{\partial \varphi^{2}} w\right) ; k_{z \varphi}=\frac{\partial}{\partial z}\left(\frac{v}{R}\right)-\frac{2}{R} \frac{\partial^{2}}{\partial \varphi \partial z} w
\end{gathered}
$$

Substitution of Eq. 10 into Eqs. 6-8 leads to the expressions for the shell displacements $<u, v, w>$ as a function of time.

\subsection{Boundary Conditions at the Fluid-Structure Interfaces}

On the internal and external shell surfaces, the particle velocities of the acoustic media in the radial direction have to be equal to the normal velocity of the shell [7]. Applying Newton's second law, the equations which describe the effect of acoustic pressure on the motion of the shell can be derived as:

$$
\begin{gathered}
\left.\frac{\partial}{\partial \mathrm{r}}\left(p_{1}^{I}+p_{1}^{R}\right)\right|_{r=R}=-\rho_{1}\left(\frac{\partial}{\partial t}+v_{z} \frac{\partial}{\partial z}\right)^{2} w \\
\left.\frac{\partial}{\partial r} p_{2}^{T}\right|_{r=R}=-\rho_{2} \frac{\partial^{2}}{\partial t^{2}} w
\end{gathered}
$$

\subsection{Solutions}

To obtain the solution of Eqs. 4 and 5, the incident wave $p_{1}^{\mathrm{I}}$ can be expressed as

$$
p_{1}^{I}=P_{0} \sum_{n=0}^{\infty} \xi_{n}(-\mathrm{i})^{n} \mathscr{I}\left(n, r k_{1 r}\right) \mathrm{e}^{\mathrm{i}\left(\omega t-k_{1 z} z-n \varphi\right)}
$$

and the reflected wave $p_{1}^{\mathrm{R}}$ :

$$
p_{1}^{R}=\sum_{n=0}^{\infty} \mathrm{A}_{n} \mathcal{H}_{2}\left(n, r k_{1 r}\right) \mathrm{e}^{\mathrm{i}\left(\omega t-k_{1 z} z-n \varphi\right)}
$$

where $P_{0}$ is the amplitude of the incident wave, $n$ is the order of the circumferential mode, $\omega=2 \pi f$ is the frequency and $A_{n}$ is the unknown constant. $\mathrm{J}$ is the Bessel function of the first kind of integer order $n(n=0,1,2,3, \ldots) . \mathcal{H}_{2}$ is the Hankel function of the second kind with integer $n$; it represents the outgoing wave from the cylinder [7].

$\xi_{n}$ is the Neumann factor given by:

$$
\xi_{n}= \begin{cases}1 & n=0 \\ 2 & n \geq 1\end{cases}
$$


and

$$
k_{1 z}=k_{1} \cos (\gamma) ; k_{1 r}=k_{1} \sin (\gamma)
$$

$k_{1}$ is the wave number for the region outside the cylinder and $\gamma$ is the incident wave angle, as shown in Fig. 1. As the acoustic wave satisfies the governing equation for the outer cavity, Eq. 5, substitution of Eqs. 18, 19 and 21 into that relation gives (for $\mathbf{V} \cdot \nabla=v_{z} \frac{\partial}{\partial z}$ ) the following expression for $k_{1}$ :

$$
k_{1}=\frac{\omega}{c_{1}}\left(\frac{1}{1+M_{1} \cos (\gamma)}\right)
$$

where $M_{1}=v_{\mathrm{z}} / c_{1}$ is the Mach number of the external flow.

The transmitted wave $p_{2}^{\mathrm{T}}$ is:

$$
p_{2}^{T}=\sum_{n=0}^{\infty} B_{n} \mathscr{H}_{1}\left(n, r k_{2 r}\right) e^{i\left(\omega t-k_{2 z} z-n \varphi\right)}
$$

where $B_{\mathrm{n}}$ is a temporally unknown complex amplitude factor; $k_{2 \mathrm{r}}$ and $k_{2 \mathrm{z}}$ are the wave numbers in the radial and circumstantial direction respectively. $\mathscr{H}_{1}$ is the Hankel function of the first kind with integer $n$. It represents the incoming wave for the cylinder.

Because the travelling waves in the cylinder and outside the cylinder are both driven by the incident-travelling wave, the wave number in the $z$ direction should match throughout the system, therefore $k_{1 \mathrm{z}}=k_{2 \mathrm{z}}$. With:

$$
k_{2}^{2}=k_{2 \mathrm{z}}^{2}+k_{2 \mathrm{r}}^{2} ; k_{2}=\frac{\omega}{c_{2}}
$$

$k_{2 \mathrm{r}}$ can be described as:

$$
k_{2 \mathrm{r}}=\sqrt{k_{2}^{2}-k_{1 \mathrm{z}}^{2}}
$$

The displacements can now be expressed by:

$$
\begin{aligned}
& u=\mathrm{i} \sum_{n=0}^{\infty} U_{n} e^{\mathrm{i}\left(\omega t-k_{1 z} z-n \varphi\right)} \\
& v=i \sum_{n=0}^{\infty} V_{n} \mathrm{e}^{\mathrm{i}\left(\omega t-k_{1 z} z-n \varphi\right)} \\
& w=\sum_{n=0}^{\infty} W_{n} \mathrm{e}^{\mathrm{i}\left(\omega t-k_{1 z} z-n \varphi\right)}
\end{aligned}
$$

where $U_{\mathrm{n}}, V_{\mathrm{n}}, W_{\mathrm{n}}$ are the unknown parameters which will be determined in the following section; they are dependent on the frequency $\omega$ and the order of the circumferential mode $n$.

\subsection{Results}

By collecting the equations of motion (6-8) and boundary condition (16-17), a system of five equations consist of $P_{0}, U_{\mathrm{n}}, V_{\mathrm{n}}, W_{\mathrm{n}}, A_{\mathrm{n}}$ and $B_{\mathrm{n}}$ is obtained. Finally the solutions for $<U_{\mathrm{n}}, V_{\mathrm{n}}, W_{\mathrm{n}}, A_{\mathrm{n}}, B_{\mathrm{n}}>$ vector are expressed by $P_{0}$ which can be eliminated later. 
Substitution of Eqs. 23 and 28 into Eq. 3 results in:

$$
W^{\mathrm{T}}=\sum_{\mathrm{n}=0}^{\infty} \frac{1}{2} \operatorname{Re}\left\{B_{\mathrm{n}} \mathscr{H}_{1}\left(n, k_{2 \mathrm{r}} r\right)\left(\mathrm{i} \omega W_{\mathrm{n}}\right) \int_{0}^{2 \pi} \cos ^{2}(n \varphi) R \mathrm{~d} \varphi\right\}, r=R
$$

where:

$$
\int_{0}^{2 \pi} \cos ^{2}(n \varphi) R \mathrm{~d} \varphi=\frac{\pi R}{\xi_{\mathrm{n}}}
$$

Eq. 29 can be simplified as:

$$
W^{\mathrm{T}}=\sum_{n=0}^{\infty} \frac{\pi R}{\xi_{\mathrm{n}}} \operatorname{Re}\left\{B_{\mathrm{n}} \mathscr{H}_{1}\left(n, k_{2 \mathrm{r}} R\right) \cdot\left(\mathrm{i} \omega W_{\mathrm{n}}\right)^{*}\right\}
$$

Finally, the transmission coefficient $\tau$ can be predicted by substituting Eqs. 2 and 31 into Eq. 1:

$$
\begin{gathered}
\tau(\gamma)=\frac{W^{\mathrm{T}}}{W^{I}}=\sum_{\mathrm{n}=0}^{\infty} \frac{\operatorname{Re}\left(B_{\mathrm{n}} \mathscr{H}_{1}\left(n, k_{2 \mathrm{r}} R\right)\left(\mathrm{i} \omega W_{\mathrm{n}}\right)^{*}\right) \rho_{1} c_{1} \pi}{\xi_{\mathrm{n}} \cos (\gamma) P_{0}^{2}} \\
\mathrm{TL}=-10 \log _{10}(\tau(\gamma))
\end{gathered}
$$

where $\tau(\gamma)$ is the transmission coefficient for a particular incident angle $\gamma$. For the diffused sound field (the wave hits the cylinder in all directions with equal sound intensity), the average sound transmission coefficient, $\tau_{\mathrm{av}}$, is given as:

$$
\begin{gathered}
\tau_{\mathrm{av}}=\int_{0}^{\frac{\pi}{2}} \tau(\gamma) \sin (2 \gamma) \mathrm{d} \gamma \\
\mathrm{TL}_{\mathrm{av}}=-10 \log _{10}\left(\tau_{\mathrm{av}}\right)
\end{gathered}
$$

\section{Introduction of the SEA and the FE\&SEA Method}

As mentioned before, the SEA is not as accurate as the FE method at low frequencies. In order to compare it with the analytical model, both the SEA and the hybrid FE\&SEA methods will be introduced here.

\subsection{The SEA Method}

For the SEA method an effective transmission model is built, consisting of the source SEA acoustic cavity, the junction area and the receiving SEA acoustic cavity, as shown in Fig. 3. Typically, the first cavity is excited by a power source or constraint and the second cavity only receives energy via the selected transmission path. 
Fig. 3 Sound transmission models by two different methods: (a) SEA method, (b) FE\&SEA
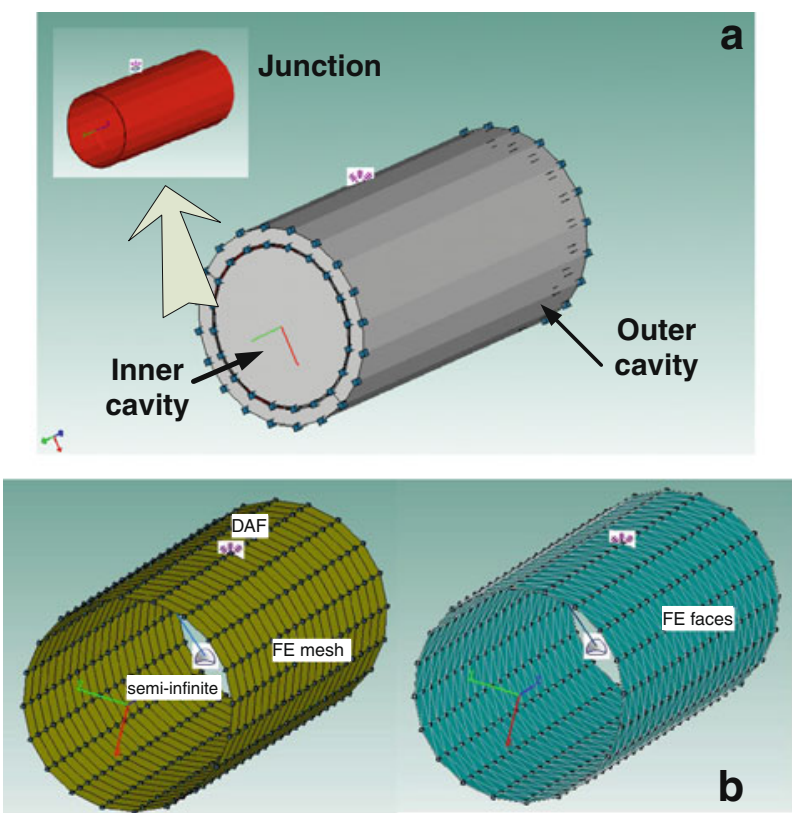

The Effective Transmission Loss is calculated using the following equation [18]:

$$
\mathrm{TL}=10 \log _{10}\left[\frac{A \omega}{8 \pi^{2} n_{1} c_{1}^{2} \eta_{2}}\left(\frac{E_{1}}{E_{2}}-\frac{n_{1}}{n_{2}}\right)\right]
$$

where $A$ is the junction area, $E_{1}$ is the energy of the source cavity, and $E_{2}$ is the energy of the receiving cavity. $n_{1}$ and $n_{2}$ are the mode density in source and receiving cavities respectively. $c_{1}$ is the acoustic wave velocity in the source cavity. $\eta_{2}$ is the loss factor in the receiving cavity.

\subsection{The Hybrid Method}

For the hybrid FE\&SEA model, TL is the transmission loss between an SEA diffused acoustic field and an SEA semi-infinite fluid separated by an FE subsystem. First the cylinder model is built and meshed in the software "Patran" and then it is imported into the software "VA one". The normal mode analysis is conducted with the external "Nastran" solver for the determination of the natural frequency. Next the FE faces are created on the existing mesh. The diffuse acoustic field (DAF) is applied on these FE faces. A semiinfinite fluid, which is a baffled acoustic half space describing the radiation of sound into an unbounded space, is connected to the cylinder. Finally, a complete hybrid model is built as shown in Fig. 3(b).

The TL is calculated by determining the net power radiated into the SEA semi-infinite fluid, and then normalizing the net power by the incident power associated with the DAF. The results depend on both the pressure difference between the DAF and SEA semi-infinite fluid and the FE faces area $A$.

The cylinder length is $10 \mathrm{~m}$ and the radius is $1.975 \mathrm{~m}$. The material properties and composites layup are the same as that used in the analytical model. The loss factor of the cylinder shell is $1 \%$ and no special noise treatment is applied on the cylinder. 
Fig. 4 Comparison between analytical and Koval's method with 45 incident angle

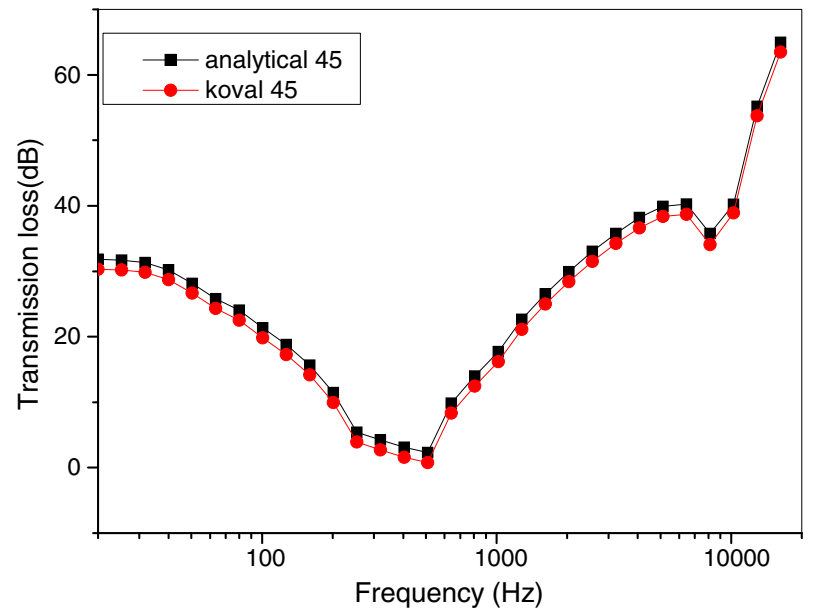

\section{Comparison of the Results Computed by the Three Methods}

In this part, the TL predicted by the analytical, SEA and FE\&SEA methods are compared to each other. For the comparison it is assumed that the external air speed is zero. First, the results of the analytical model are compared with that of Koval's analytical model [4]. As shown in Fig. 4, for an incident angle of $45^{\circ}$ there is little difference between the results provided by these two models.

Second, the results generated by the three methods are compared under the diffused acoustic field. For the analytical method, Eqs. 34 and 35 are used to obtain the TL of the DAF by averaging the TL of different incident angles.

Figure 5 shows the transmission loss computed by the analytical model, the SEA model and the hybrid FE\&SEA respectively. For the hybrid model, the FE method is applied in the low frequency range $(20 \sim 800 \mathrm{~Hz})$ and SEA is used for the middle and high frequencies. It can be seen that all of them have two minimum values, one is between $400 \sim 600 \mathrm{~Hz}$ and the other is between $4,000 \sim 6,000 \mathrm{~Hz}$. The first one corresponds to the ring frequency $f_{\mathrm{R}}$ and

Fig. 5 TL of composites cylinder predicted by the three methods

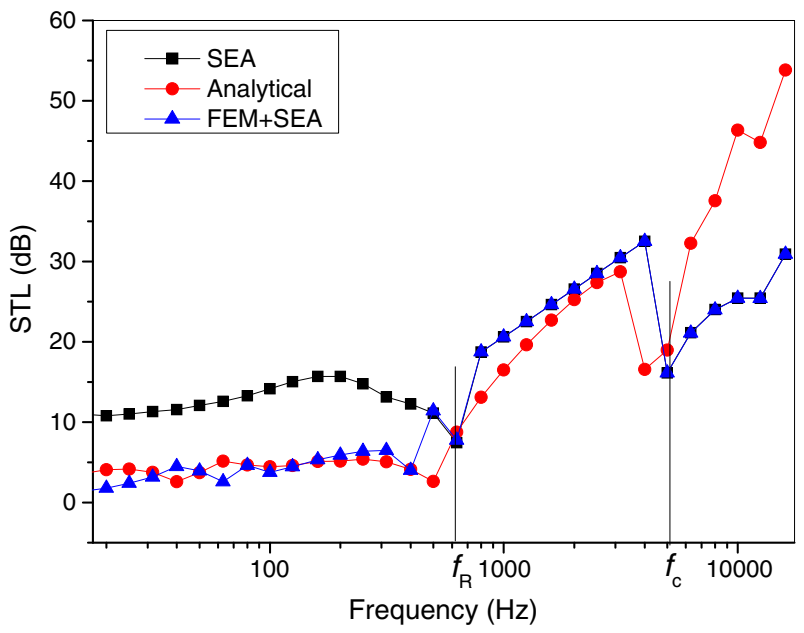


the other to the critical frequency $f_{\mathrm{c}}$. Some equations to estimate the ring frequency and critical frequency are [19]:

$$
\begin{gathered}
f_{\mathrm{R}}=\frac{1}{2 \pi R} \sqrt{\frac{E_{\varphi} \alpha}{\rho\left(\alpha-\mu_{12}^{2}\right)}} \\
f_{c}=\frac{c^{2}}{2 \pi} \sqrt{\frac{I}{D_{11}}}
\end{gathered}
$$

where $\alpha=E_{\mathrm{z}} / E_{\varphi}, E_{\mathrm{z}}$ and $E_{\varphi}$ are the composites modulus of elasticity, $D_{11}$ is the first element of the matrix $\mathbf{D}$ in Eq. 13, $c$ is the sound speed through the cylinder, and $I$ can be obtained by Eq. 9. Based on Eqs. 37 and 38 and mechanical properties of the laminate, the results $f_{\mathrm{R}}=541 \mathrm{~Hz}$ and $f_{\mathrm{c}}=4,626 \mathrm{~Hz}$ are readily obtained. They are comparable with the results in the Fig. 5.

The middle region between them is mass controlled. The panel vibration and noise transmission are mainly controlled by the stiffness below $f_{\mathrm{R}}$, and mainly controlled by the damping above $f_{\mathrm{c}}$. At the ring frequency, the longitudinal wavelength in the cylindrical shell is equal to the circumference wavelength and an axisymmetric resonance occurs [15]. At the critical frequency, the wavelength of the forced flexural wave in the fuselage structure equals the wavelength of the bending acoustic wave. When this takes place, the intensity of the transmitted wave approaches the intensity of the incident wave. The bending waves become "acoustically fast" and the panel radiates from its whole area instead of just its edges or corners [20].

The three curves in Fig. 5 show the same trend as the frequency increases. The positions of $f_{\mathrm{R}}$ and $f_{\mathrm{c}}$ become close to each other. It can be seen that the SEA results are higher than the analytical and the FE\&SEA results at low frequencies. At high frequencies, the results of the analytical model show a higher TL. This is probably due to the thin shell theory assumption and the absence of transverse shear. Kamran Daneshjou [8] studied the effect of transverse shear using the first order shell theory and found that it increased the TL prediction (Fig. 10 in his paper).

\section{Study on Composite Cylinders by the SEA Model}

The human hearing ability is in the range $20-20,000 \mathrm{~Hz}$, but the hearing sensibility of the human ear is not constant over the audible frequency range. Figure 6 shows that the ear is less sensitive to low frequencies and very sensitive to frequencies around 3,000-4,000 Hz.

In the following parts, because the SEA models are more convenient to predict the TL and human's ear is less sensitive to noise at low frequencies, the SEA method is used to study the influence parameters on the TL of composites cylinders. The parameters include the laminate layup, the fiber material, the cylinder dimensions and the structure morphology (sandwich or stiffened structure).

\subsection{Effect of the Laminate Layup}

One of the advantages of composite materials over isotropic materials is their ability for structural tailoring. In order to evaluate the effect the layup has on the transmission loss, four different arrangements are here chosen (as shown in Table 1). 
Fig. 6 Equal loudness curves [21]

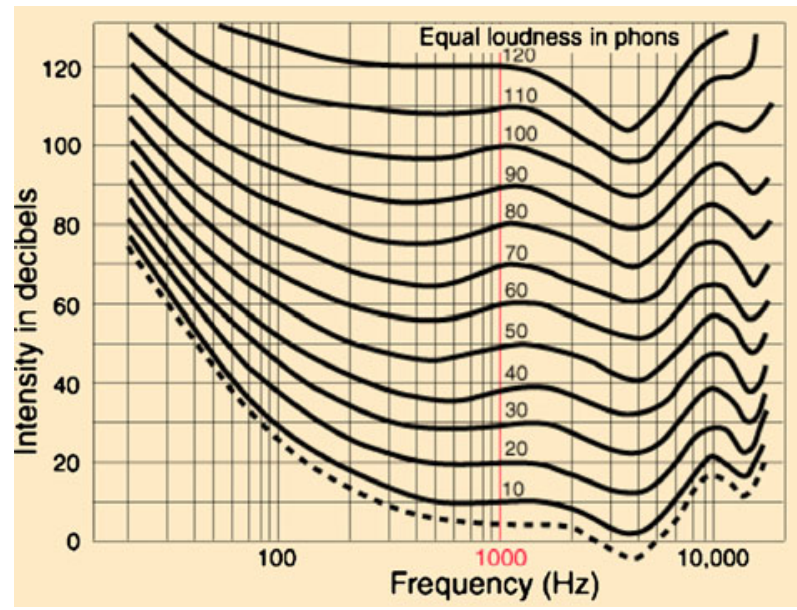

For the layup $[45 /-45 / 45 /-45 / 45]_{\mathrm{s}}$, an increase of the $45^{\circ}$ ply improves the shear stiffness $G_{12}$ but reduces the extensional stiffness $E_{\varphi}$ and bending stiffness $D_{11}$, leading to a downward shift of the ring frequency and a upward shift of the critical frequency. For the layers $[0 / 10 / 20 /$ $30 / 40 / 50 / 60 / 70 / 80 / 90]$ and $[0 / 90]_{5}$, the absolute value of bending-extensional coupling $B$ is not zero. This layup can dissipate more vibration energy in its own structure, and thus the TL of these two is larger than that of the others outside the mass-controlled range. For the layup $[0 / 90 / 45 /-45 / 0]_{\mathrm{s}}$, its TL is the lowest outside the mass-controlled range (Fig. 7).

\subsection{The Effect of Material}

The material choice is an important issue for composite fuselage design. Thus three different kinds of composites are here selected to evaluate their influence on the TL. For a

Table 1 Mechanical properties of different layups

\begin{tabular}{|c|c|c|c|c|c|}
\hline Layup & $E_{\mathrm{z}}[\mathrm{Pa}]$ & $E_{\varphi}[\mathrm{Pa}]$ & $G_{12}[\mathrm{~Pa}]$ & B & $\mathbf{D}_{11}$ \\
\hline$[45 /-45 / 45 /-45 / 45]_{\mathrm{s}}$ & $1.77 \mathrm{E} 10^{\mathrm{a}}$ & $1.77 \mathrm{E} 10$ & $5.62 \mathrm{E} 10$ & $\left(\begin{array}{lll}0 & 0 & 0 \\
0 & 0 & 0 \\
0 & 0 & 0\end{array}\right)$ & 11.5 \\
\hline$[0 / 90 / 45 /-45 / 0]_{\mathrm{s}}$ & $1.08 \mathrm{E} 11$ & $6.78 \mathrm{E} 10$ & $2.54 \mathrm{E} 10$ & $\left(\begin{array}{lll}0 & 0 & 0 \\
0 & 0 & 0 \\
0 & 0 & 0\end{array}\right)$ & 22.9 \\
\hline$[0 / 90]_{5}$ & $1.14 \mathrm{E} 11$ & $1.14 \mathrm{E} 11$ & $4.80 \mathrm{E} 9$ & $\left(\begin{array}{llll}-9 E 3 & 0 & 0 \\
0 & 9 E 3 & 0 \\
0 & 0 & 0 & \end{array}\right)$ & 20.9 \\
\hline$[0 / 10 / 20 / 30 / 40 / 50 / 60 / 70 / 80 / 90]$ & $8.42 \mathrm{E} 10$ & $8.42 \mathrm{E} 10$ & $2.49 \mathrm{E} 10$ & $\left(\begin{array}{ccc}-3.8 E 4 & 0 & -5.3 E 3 \\
0 & 3.8 E 4 & 5.37 E 3 \\
-5.3 E 3 & 5.37 E 3 & 0\end{array}\right)$ & 19.5 \\
\hline
\end{tabular}

\footnotetext{
${ }^{\mathrm{a}}$ E10 denotes $10^{10}$
} 
Fig. 7 Comparison among different lay up of composite plies

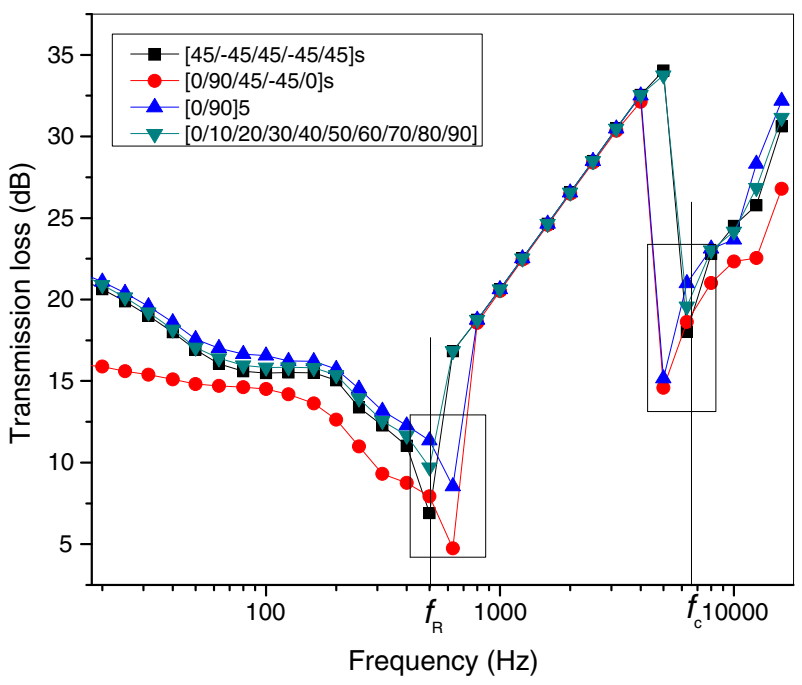

representative comparison, the models with different kinds of materials have the same layup and thickness. The three different kinds of materials are shown in Table 2.

As shown in Fig. 8, the curve which represents the Fiberglass/Epoxy composites has the largest TL at the mass controlled range. This is because of the higher density of Fiberglass. In addition, the Fiberglass/epoxy composite shows a larger mass controlled range due to its lower modulus of elasticity and bending stiffness referring to Eqs. 37 and 38. At low frequencies, Graphite/Epoxy has the larger TL because of the highest stiffness of Carbon fiber.

\subsection{The Effect of Radius and Length}

In the SEA methodology, the cylinder is regarded as a single subsystem. To investigate the effect of the geometry on the TL, the radius and length of the cylinder are varied. Other parameters like materials properties and atmospheric conditions are kept constant.

Figures 9 and 10 show the effect of $L$ and $R$ on the cylinder TL respectively. It can be seen in Fig. 9 that the length $L$ has little influence on the TL. However, as shown in Fig. 10, an increase of the radius $R$ leads to a decrease of the ring frequency. This can be explained by Eq. 37. Moreover, the cylinder with smaller $R$ exhibits a larger TL at low frequencies. Finally, it is worth noting that $R$ has no effect on the TL at high frequencies.

Table 2 Properties of three kinds of materials

\begin{tabular}{llll}
\hline & Graphite/Epoxy & Fiberglass/Epoxy & Aramid/Epoxy \\
\hline Density[kg/m $\left./ \mathrm{m}^{3}\right]$ & 1,600 & 1,900 & 1,500 \\
$E_{1}[\mathrm{~Pa}]$ & $2.21 \mathrm{E} 11$ & $5.6 \mathrm{E} 10$ & $7.6 \mathrm{E} 10$ \\
$E_{2}[\mathrm{~Pa}]$ & $6.9 \mathrm{E} 9$ & $1.3 \mathrm{E} 10$ & $5.5 \mathrm{E} 9$ \\
$v_{12}$ & 0.25 & 0.26 & 0.34 \\
$G_{12}[\mathrm{~Pa}]$ & $4.8 \mathrm{E} 9$ & $4.2 \mathrm{E} 9$ & $2.3 \mathrm{E} 9$ \\
\hline
\end{tabular}


Fig. 8 Comparison among three different kinds of materials

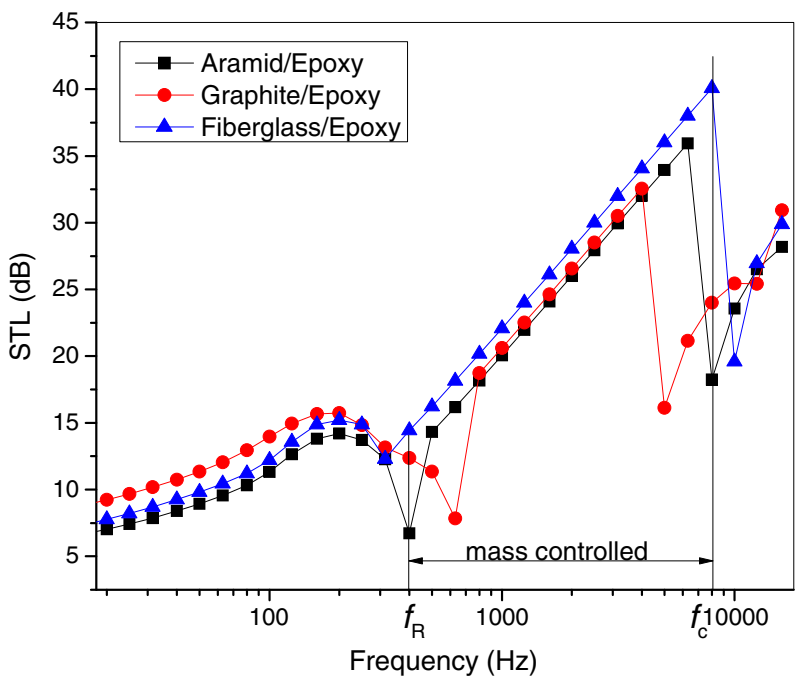

\subsection{Effect of the Structure Morphology}

The stiffened and sandwich structure are the most commonly applied configurations in aerospace because of their mechanical efficiency. In contrast to their mechanical properties, their sound insulation capability is seldom studied. Therefore, in this section, firstly some representative sandwich structures are studied and then compared to classical laminates. Secondly, a representative sandwich panel is compared to its stiffened counterpart.

\subsubsection{Comparison Between the Sandwich Panels and the Laminates}

In order to investigate whether the sandwich structure is a good choice in terms of sound insulation, an anisotropic foam is added inbetween the laminate facings. The effect of core

Fig. 9 TL of cylinder with different length $(L)$

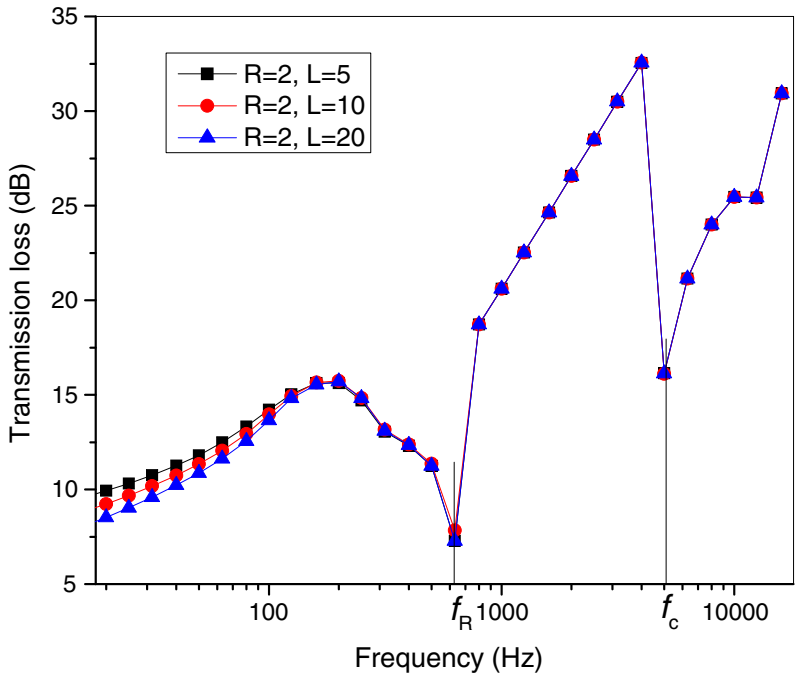


Fig. 10 TL of cylinder with different $\operatorname{radius}(\mathrm{R})$

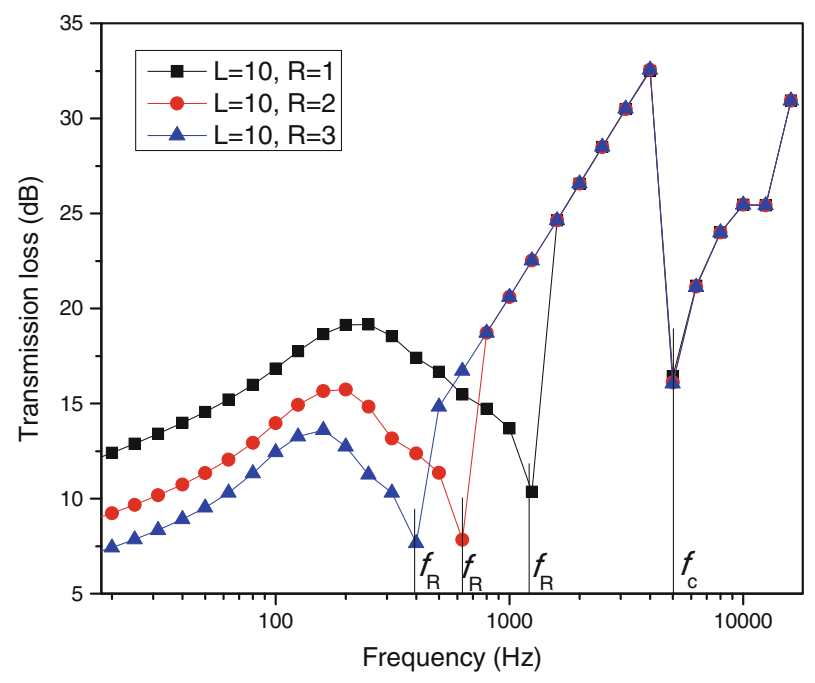

thickness and structural layout (sandwich and monolithic) are also investigated. The core properties are:

$$
E_{c}=350 \mathrm{MPa}, G_{c}=150 \mathrm{Mpa}, v_{c}=0.31, \rho_{c}=120 \mathrm{~kg} / \mathrm{m}^{3}
$$

The core thicknesses $t_{c}$ are $10 \mathrm{~mm}, 20 \mathrm{~mm}$ and $30 \mathrm{~mm}$ respectively.

As shown in Fig. 11, unlike laminated composites, the sandwich structures show very shallow dips in the TL-Frequency curves except for the thinnest panel with $t_{\mathrm{c}}=10 \mathrm{~mm}$. This phenomenon is comparable to Figs. (5-8) of [6]. The absence of dips is caused by the much higher bending stiffness of sandwich panels when compared to laminates, as shown in Table 3. For the sandwich structure, the increased bending stiffness causes $f_{\mathrm{c}}$ to shift towards low frequencies; in this region the SEA method cannot accurately predict the dips.

Fig. 11 Effect of the core thickness on the TL

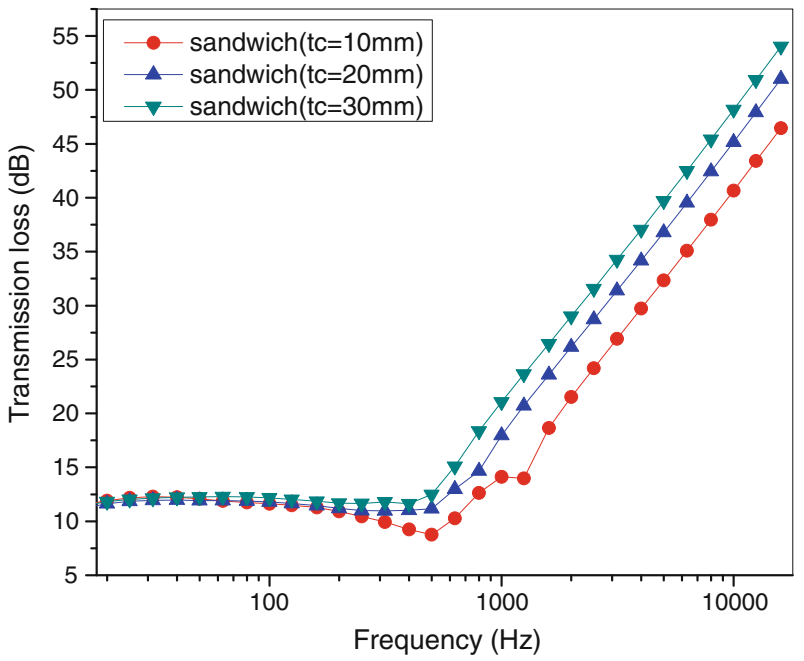

Springer 
Table 3 Bending stiffness $S_{\mathrm{b}}$ and critical frequency $f_{\mathrm{c}}$

\begin{tabular}{lllll}
\hline & 10-layered laminates & Sandwich $t_{\mathrm{c}}=10 \mathrm{~mm}$ & Sandwich $t_{\mathrm{c}}=20 \mathrm{~mm}$ & Sandwich $t_{\mathrm{c}}=30 \mathrm{~mm}$ \\
\hline$S_{\mathrm{b}}[\mathrm{Nm}]$ & 23 & 7,240 & 25,125 & 53,789 \\
$f_{\mathrm{c}}[\mathrm{Hz}]$ & 4,626 & 552 & 323 & 238 \\
\hline
\end{tabular}

The bending stiffness of the sandwich is estimated by: $S_{\mathrm{b}}=(1 / 2) E_{\mathrm{f}} t_{\mathrm{f}}\left(t_{\mathrm{f}}+t_{\mathrm{c}}\right)^{2}$

The raised bending stiffness also results in the less distinct dips for the TL of sandwich panels [22]. Furthermore, increase of the core thickness leads to a larger TL at high frequencies but has little effect at low frequencies. The TL increase from $t_{\mathrm{c}}=10 \mathrm{~mm}$ to $t_{\mathrm{c}}=$ $20 \mathrm{~mm}$ is larger than that from $t_{\mathrm{c}}=20 \mathrm{~mm}$ to $t_{\mathrm{c}}=30 \mathrm{~mm}$.

In Fig. 12, in order to study the influence of the laminate thickness on the TL, the TL of a 10-layered laminate is compared to that of a 20-layered laminate (double thickness of the 10layered). It can be seen that an increase of the thickness leads to an overall TL enhancement. In addition, a forward shift of $f_{\mathrm{R}}$ and a backward shift of $f_{\mathrm{c}}$ do take place. For investigating the core effect, a sandwich panel with $t_{\mathrm{c}}=21.2 \mathrm{~mm}$ is chosen with the same weight as the 20layered laminates. As shown in Fig. 14, the TL of the sandwich structure is lower than that of the 20-layered laminate at the range $20 \sim 2,000 \mathrm{~Hz}$. However, it is much larger than the TL of the 20-layered laminate above $2,000 \mathrm{~Hz}$; it attributes to the high inherent damping ability of the viscoelastic core. At 3,000 4,000 Hz where humans are most sensitive to sound (Fig. 6), the laminates provide poor sound insulation because of the second dips (Fig. 12). Nevertheless, the sandwich structure presents a much better sound insulation at this range.

\subsubsection{Comparison Between Sandwich and Stiffened Structure}

In this section, a stiffened panel will be compared to a sandwich panel in terms of sound insulation. For the stiffened panel the skin is also made of 10-layered Carbon/Epoxy composite, and the stiffener material is aluminum (only isotropic materials are allowed to

Fig. 12 Comparison between laminates and sandwich with the same weight

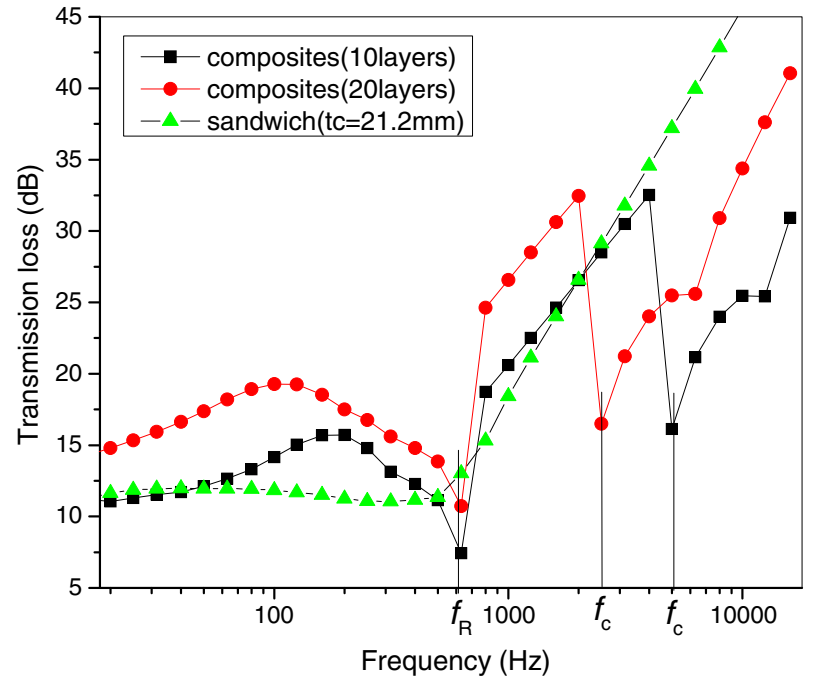


Fig. 13 The dimension of stiffeners and their pitch

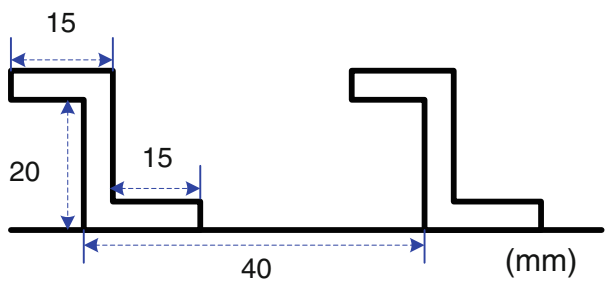

be chosen as a stiffener in the "VA one" software). The Z stiffeners dimensions and their pitches are shown in Fig. 13; they are aligned in the axial direction of the cylinder. For the sandwich panel, the core thickness is $28 \mathrm{~mm}$ in order to have the same weight with the stiffened structure. As a reference, the TL of a 20-layered laminate is also added in Fig. 14. However, it should be noted here that the 20-layered laminate has less weight than the stiffened and sandwich panels.

As shown in Fig. 14, the stiffened structure has lower TL than the sandwich structure, especially at frequencies above $1,000 \mathrm{~Hz}$. The TL of the stiffened panel is even lower than the TL of the non-stiffened laminate. L.A. Krakers [14] tested the sound transmission of three cylinders including one non-stiffened cylinder, and two cylinders stiffened by 6 stringers and 12 stringers respectively. The results show that the non-stiffened cylinder has larger TL than the two stiffened panels. Another acoustic transmission measurement on a stiffened fuselage demonstrates that the window area has larger TL than the stringer area [23]. This phenomenon takes place because the excitation efficiency of the stiffened structure is higher than that of the monolithic and the sandwich structures. Furthermore, for the stiffened cylinder, the mass-controlled range is not as apparent as that for the laminate. In addition, it presents more dips than the laminate and sandwich structure. An overview of the simulation results is given in Table 4.

Fig. 14 Comparison between the stiffened structure and sandwich structure

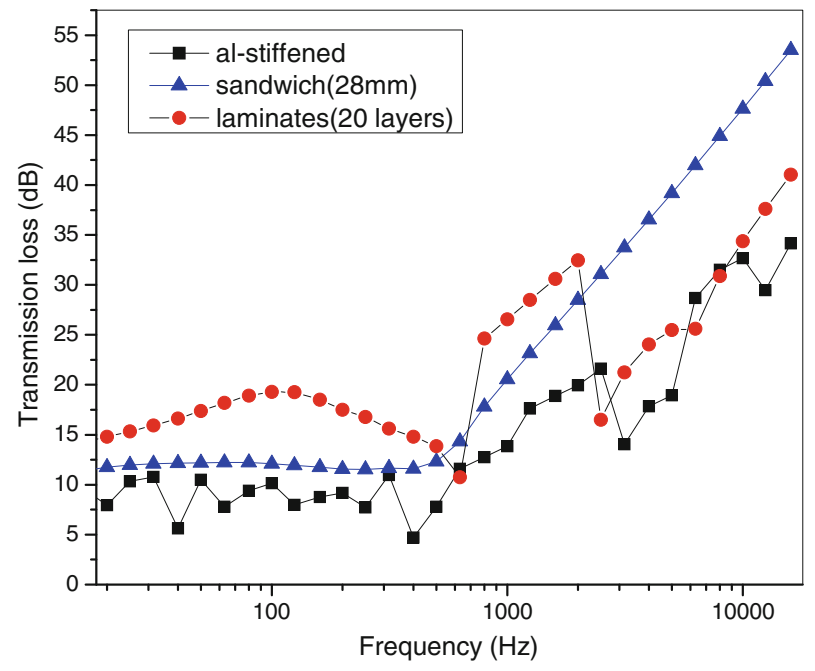

Springer 
Table 4 Summary of the transmission loss of different structure types

\begin{tabular}{llll}
\hline & Non-stiffened cylinder & Sandwich cylinder & Stiffened cylinder \\
\hline TL at $20 \sim 2,000 \mathrm{~Hz}$ & Highest & Middle & Lowest \\
TL at $2,000 \sim 2,000 \mathrm{~Hz}$ & Middle & Highest & Lowest \\
Dips of the TL curve & 2 or 3 & Very few & More than 4 \\
\hline
\end{tabular}

\section{Conclusion}

The investigation presented here focuses on the prediction of sound transmission loss of a fuselage by different methods. The fuselage is modeled as a cylindrical composite shell. First, an analytical method is given to predict the TL of an infinite-length cylinder. Second, the statistic energy analysis (SEA) method and a hybrid finite element (FE)\&SEA model are applied for predicting the cylinder TL. Third, the three methods are compared to each other. Fourth, although the hybrid method provides more accuracy compared with the SEA model at low frequencies, the SEA model is finally chosen for studying the influence of geometric and material parameters have on the TL of cylinders. The reason for this model choice lies in its computational efficiency and low degree of complexity. The following parameters are studied:

- Laminate layup

- Fiber material

- Cylinder dimensions

- Structure morphology

It is found that for a typical laminate asymmetric layups do amplify bendingextensional coupling, which in turn enhances the TL. A thickness increase of the $45^{\circ}$ layers leads to a downward shift of the ring frequency and an upward shift of the critical frequency. Furthermore, Fiberglass/Epoxy laminates exhibit the largest TL in the mass-controlled region and the damping-controlled region as compared to Graphite/ Epoxy and Aramid/Epoxy laminates. Next, in terms of the geometry, the length of the considered cylinders has little influence on its TL, but on the other hand, increasing the radius decreases the ring frequency and the TL at low frequencies. Finally, for the sandwich structure, an increase of the core thickness leads to a backward shift of the critical frequency and a large TL enhancement at high frequencies. Based on the nonstiffened laminate, the stiffened panel provides a lower TL but the sandwich panel improves the TL dramatically at frequencies above $2,000 \mathrm{~Hz}$.

In conclusion, it is convenient to predict the TL of fuselages with the SEA method mentioned in this paper. The TL predicted by this method is well-comparable to the one computed by the analytical method. However, it is too simple to model a real fuselage as a cylinder, thus the fuselage with windows, floors and interior insulation blankets will be studied as part of future research, including experimental verification.

Open Access This article is distributed under the terms of the Creative Commons Attribution Noncommercial License which permits any noncommercial use, distribution, and reproduction in any medium, provided the original author(s) and source are credited. 


\section{References}

1. Davis, G.W., Sakata, I.F.: Design considerations for composite fuselage structure of commercial transport aircraft. NASACR 159296, Lockheed-California company (1981)

2. Koval, L.R.: On sound transmission into a thin cylindrical shell under flight conditions. J. Sound Vib. 48, 265-275 (1976)

3. Koval, L.R.: On sound transmission into an orthotropic shell. J. Sound Vib. 63, 51-59 (1979)

4. Koval, L.R.: Sound transmission into a laminated composite cylindrical shell. J. Sound Vib. 71, 523-530 (1980)

5. ESDU07001: Noise transmission into aircraft cabins (2007)

6. Tang, Y.Y., Robinson, J.H., Silcox, R.J.: Sound transmission through a cylindrical sandwich shell with honeycomb core. NASA (1996)

7. Daneshjou, K., Nouri, A., Talebitooti, R.: Sound transmission through laminated composite cylindrical shells using analytical model. Arch. Appl. Mech. 77, 363-379 (2007)

8. Daneshjou, K., Nouri, A., Talebitooti, R.: Analytical model of sound transmission through laminated composite cylindrical shells considering transverse shear deformation. Appl. Math. Mech. 29, 1165$1177(2008)$

9. Arjunan, R.: Vibroacoustic parametric analysis of honeycomb composite fuselage for improved transmission loss, Master Thesis, Vichita State University (2007)

10. Li, Z.: Vibration and acoustical properties of sandwich composite materials, PhD Thesis, Auburn University (2006)

11. ESDU99009: An introduction to statistical energy analysis (1999)

12. Ghinet, S., Atalla, N., Osman, H.: The transmission loss of curved laminates and sandwich composite panels. J. Acoust. Soc. Am. 118, 774 (2005)

13. Ford, R.D., Lord, P., Walker, A.W.: Sound transmission through sandwich constructions. J. Sound Vib. 5, 9-21 (1967)

14. Krakers, L.A.: Parametric fuselage design, PhD Thesis, Delft University of Technology (2009)

15. Fahy, F.J., Gardonio, P.: Sound and structural vibration: radiation, transmission and response, 2nd edn. Academic, Oxford (2007)

16. Adams, R.C., Advani, S.: Composites-asm handbook volume 21 (2001)

17. Soedel, W.: Vibrations of shells and plates. Marcel Dekker, New York (1993)

18. ESI-group: Va one 2007 user guide (2007)

19. Blaise, C.L.A., Gotteland, M., Barbe, M.: On sound transmission into an orthotropic infinite shell: comparison with Koval's results and understanding of phenomena. J. Sound Vib. 150, 233-243 (1991)

20. ESDU02020: An introduction to aircraft noise (2002)

21. http://hyperphysics.phy-astr.gsu.edu/hbase/sound/eqloud.html\#c2 (Accessed 21 September, 2009)

22. Nilsson, A.C.: Wave propagation in and sound transmission through sandwich plates. J. Sound Vib. 138, 73-94 (1990)

23. Wal, H.; Nilsson, A.C.: Sound-transmission measurements on composite and metal fuselage panels for different boundary conditions, NLR-TP-2005-570. National Aerospace Laboratory (2005) 